G. D. Dikshit, Department of Mathematics, University of Auckland, Private Bag 92019, New Zealand. e-mail: dixit@math.auckland.ac.nz

\title{
A NOTE ON THE ABSOLUTE SUMMABILITY OF FOURIER SERIES BY BOSANQUET-LINFOOT METHOD
}

\begin{abstract}
In this note we show that a theorem due to L.I. Holder on the absolute summability of Fourier series by the Bosanquet-Linfoot method $|\alpha, \beta|$, can be improved upon. Our theorem then provides a better refinement to the classical theorem of Bosanquet on summability $|C, \gamma|$ of Fourier series.
\end{abstract}

\section{Introduction}

With intentions to make refinements on results familiar on Cesàro summability of Fourier series, Bosanquet and Linfoot $[4,5]$ introduced the concept of a new summability method that they termed as the method $(\alpha, \beta)$ :

Definition 1. Let $\alpha>0, \beta$ a real number, or $\alpha=0$, and $\beta \geq 0$. A series $\sum u_{n}$ is said to be summable $(\alpha, \beta)$ to a sum $s$, if

$$
\lim _{\omega \rightarrow \infty} \sum_{n<\omega} B\left(1-\frac{n}{\omega}\right)^{\alpha}\left(\log \frac{C}{1-\frac{n}{\omega}}\right)^{-\beta} u_{n}=s
$$

for all sufficiently large values of $C$ and $B=(\log C)^{\beta}$. The method $(0,0)$ is thus defined as the convergence.

Key Words: Bosanquet-Linfoot method, Nevanlinna method, absolute summability of Fourier series

Mathematical Reviews subject classification: $42 \mathrm{~A} 28$

Received by the editors March 13, 2001 
The absolute summability method $|\alpha, \beta|$ was introduced much later by Boyer and Holder [6]. Indeed the series $\sum u_{n}$ is said to be absolutely summable $(\alpha, \beta)$, or just summable $|\alpha, \beta|$, if

$$
\int_{A}^{\infty}\left|\frac{d}{d \omega}\left(\sum_{n<\omega} B\left(1-\frac{n}{\omega}\right)^{\alpha}\left(\log \frac{C}{1-\frac{n}{\omega}}\right)^{-\beta} u_{n}\right)\right| d \omega<\infty
$$

where $A>0$ and the parameters $\alpha, \beta$ and the constants $B$ and $C$ are as for the method $(\alpha, \beta)$. The method $|0,0|$ is again taken to define the absolute convergence.

We may note that for $\beta=0$ the method $(\alpha, \beta)$ is the same as the Riesz method $(R, n, \alpha)$. Similarly, for $\beta=0$ the method $|\alpha, 0|$ is the Riesz method $|R, n, \alpha|$. As these Riesz methods are equivalent to the corresponding Cesàso methods of 'order' $\alpha$, results for summability $(\alpha, \beta)$ and summability $|\alpha, \beta|$ relate to results on Cesàso summability.

For the method $|\alpha, \beta|$, Boyer and Holder (loc.cit.) gave the following consistency results:

$|\alpha, \beta| \subset\left|\alpha^{\prime}, \beta^{\prime}\right|$ where either (i) $\alpha^{\prime}>\alpha$ (and any $\beta$ and $\beta^{\prime}$ ), or (ii) $\alpha^{\prime}=\alpha$ and $\beta^{\prime} \geq \beta$.

(As usual, over here by ' $|T|$ ' we also mean the vector space of series summable by the method $|T|$.)

In particular, then $|\alpha, \beta| \subset\left|C, \alpha^{\prime}\right|$, for $\alpha^{\prime}>\alpha$ and $\beta$ any real number.

\section{Notation}

Let $f \in L(-\pi, \pi)$ and be a periodic function of period $2 \pi$ and let

$$
f(x) \sim \frac{1}{2} a_{0}+\sum_{1}^{\infty}\left(a_{n} \cos n x+b_{n} \sin n x\right) \equiv \sum_{0}^{\infty} A_{n}(x) .
$$

Let

$$
\phi(t)=\frac{1}{2}\{f(x+t)+f(x-t)\}
$$

and let

$$
\Phi_{0}(t)=\phi(t)
$$

and for $\alpha>0$

$$
\begin{aligned}
\Phi_{\alpha}(t) & =\frac{1}{\Gamma(\alpha)} \int_{0}^{t}(t-u)^{\alpha-1} \phi(u) d u \\
\phi_{\alpha}(t) & =\Gamma(\alpha+1) t^{-\alpha} \Phi_{\alpha}(t), \quad \alpha \geq 0 .
\end{aligned}
$$


The study of $|\alpha, \beta|$-summability of Fourier series was taken up by Holder[9] and Patra[13] rather simultaneously. Holder gave the following theorem:

Theorem A. [9]. Let $\alpha \geq 0$ and suppose $\phi_{\alpha}(t) \in B V(0, \pi)$. Then the Fourier series $\sum A_{n}(x)$ is summable $|\alpha, \beta|$, where (i) $\beta>1$ if $\alpha=0$ and (ii) $\beta>2$ if $\alpha>0$.

The case $\alpha=0, \beta>1$ was given independently by Patra [13].

In view of the inclusion relations between the method $|\alpha, \beta|$ and the Cesàso method $|C, \gamma|$, this theorem improves upon the classical result of Bosanquet:

Theorem B. $[1,2]$. Let $\gamma>\alpha \geq 0$. Then $\phi_{\alpha}(t) B V(0, \pi) \Rightarrow \sum A_{n}(x) \in|C, \gamma|$.

We note that in Theorem A the parameter $\beta$ has two different ranges associated with different values of $\alpha$ : (i) $\beta>1$ if $\alpha=0$ and (ii) $\beta>2$ for $\alpha>0$. A natural question arises whether it is possible to have ' $\beta>1$ ' for all $\alpha, \alpha \geq 0$, in Theorem A. Here we show that the answer to this question is in affirmative. However, we need to recall some further concepts for this.

\section{Nevanlinna Summability}

Nevanlinna [12] and Moursund [10,11] studied a series-to-function transformation, which we call as the method $N\left(q_{\delta}\right)$, with intentions to improve upon some known results on Cesàro summability of Fourier series and differentiated Fourier series. Ray and Samal [15], Samal [16] and Dikshit [8] have studied the corresponding absolute summability method $\left|N\left(q_{\delta}\right)\right|$ :

Definition 2. Let $\sum u_{n}$ be a given series and let $F(\omega)=\sum_{n<\omega} u_{n}$. Let $q_{\delta}=q_{\delta}(t)$ be defined for $0 \leq t<1$. The $N\left(q_{\delta}\right)$-transform $N\left(F, q_{\delta}\right)$ of $F$ is defined by

$$
N\left(F, q_{\delta}\right)(\omega)=\int_{0}^{1} q_{\delta}(t) F(\omega t) d t .
$$

The series $\sum u_{n}$ is said to be summable by the method $N\left(q_{\delta}\right)$ to a sum $s$ if

$$
\lim _{\omega \rightarrow \infty} N\left(F, q_{\delta}\right)(\omega)=s .
$$

$\sum u_{n} \in\left|N\left(q_{\delta}\right)\right|$, if for some $A>0$,

$$
\int_{A}^{\infty}\left|\frac{d}{d \omega} N\left(F, q_{\delta}\right)(\omega)\right|<\infty .
$$

The parameter $\delta, \delta \geq 0$, controls the comparative strength of the methods $N\left(q_{\delta}\right)$ and $\left|N\left(q_{\delta}\right)\right|$. 
The $\left|N\left(q_{\delta}\right)\right|$-summability of Fourier series has been done rather extensively quite recently in [8].

For the regularity of the method, and for other requirements, the kernel function $q_{\delta}$ for the method $\left|N\left(q_{\delta}\right)\right|$ is supposed to satisfy the following hypotheses:

(1) $\int_{0}^{1} q_{\delta}(t) d t=1$.

(2) In the case $0 \leq \delta<1, q_{\delta}(t)$ is increasing for $0<t<1$.

(3) In the case $\delta \geq 1$, let $p=[\delta]$, the integral point of $\delta$. It is taken that $q_{\delta}$ satisfies the following (3a)-(3d):

(3a) $q_{\delta}(t)$ is non-increasing for $0<t<1$,

(3b) $\left(\frac{d}{d t}\right)^{p-1} q_{\delta}(t) \in A C[0,1]$

(3c) $\left.\left(\frac{d}{d t}\right)^{k} q_{\delta}(t)\right]_{t=1}=0, k=0,1,2, \cdots,(p-1)$

(3d) $(-1)^{p}\left(\frac{d}{d t}\right)^{p} q_{\delta}(t) \geq 0$, and is increasing.

Also, for $\delta \geq 0, p=[\delta]$,

(4) $\frac{Q_{\delta}(t)}{t^{\delta-p+1}} \in L(0,1)$, where $Q_{\delta}(t)=\int_{1-t}^{1} q_{\delta}^{(p)}(x) d x$.

In [8] the following theorem on the absolute Nevanlinna summability of Fourier series has been proved:

Theorem C. [8]. Let $\alpha \geq 0$ and let the function $q_{\alpha}$ satisfy the conditions (1)-(4) with $\delta=\alpha$. If $\phi_{\alpha}(t) \in B V(0, \pi)$ then $\sum A_{n}(x) \in\left|N\left(q_{\alpha}\right)\right|$.

The case $\alpha=0$ is due to Ray and Samal [15].

\section{The Main Result}

We give the following theorem:

Theorem 4.1.. Let $\alpha \geq 0$. If $\phi_{\alpha}(t) \in B V(0, \pi)$ then the series $\sum A_{n}(x)$ is summable $|\alpha, \beta|$, for all $\beta>1$. 
Remark 4.2.. 1. Recall that (i) $|\alpha, \beta| \subset\left|\alpha^{\prime}, \beta^{\prime}\right|$, for $\alpha^{\prime}>\alpha$ and any $\beta$ and $\beta^{\prime}$, and that (ii) $|C, \alpha|=|\alpha, 0|$. In view of these relations we may note that the theorem given above presents a refinement to Theorem B of Bosanquet. It may be remarked that most of the familiar theorems given for various other absolute summability methods tend to produce extensions of Theorem B, rather than bring improvements on it.

2. Very recently Chandra and Karanjgaokar[7] have given an alternate proof for the case $\alpha=1$ of the theorem.

Proof of Theorem 4.1.. We deduce the theorem from Theorem C.

Let $G_{\alpha, \beta}(u)=B u^{\alpha}\left(\log \frac{C}{u}\right)^{-\beta}$, for $0<u \leq 1$ and let $G_{\alpha, \beta}(0)=0$. Then in order that $\sum u_{n}$ be summable $|\alpha, \beta|$, one needs to show that

$$
\int_{A}^{\infty}\left|\sum_{n<\infty} G_{\alpha, \beta}^{\prime}\left(1-\frac{n}{\omega}\right) n u_{n}\right| \frac{d \omega}{\omega^{2}}<\infty,
$$

for some $A>0$. However, $\sum u_{n} \in\left|N\left(q_{\alpha}\right)\right|$, if and only if,

$$
\int_{A}^{\infty}\left|\sum_{n<\omega} q_{\alpha}\left(\frac{n}{\omega}\right) n u_{n}\right| \frac{d \omega}{\omega^{2}}<\infty,
$$

for some $A>0$. Thus a proof for the theorem is done if we have that $q_{\alpha}(t)=$ $G_{\alpha, \beta}^{\prime}(1-t), \quad 0 \leq t<1$ and $q_{\alpha}(1)=G_{\alpha, \beta}^{\prime}(0)=0$ meets the hypotheses on $q_{\alpha}$ in Theorem C. However, we note that for $0<t<1$,

(i) $G_{0, \beta}^{\prime}(1-t)=\frac{B}{(1-t)} \beta\left(\log \frac{C}{1-t}\right)^{-\beta-1}$,

(ii) for $\alpha>0, G_{\alpha, \beta}^{(k)}(1-t)=B(1-t)^{\alpha-k}\left(\log \frac{C}{(1-t)}\right)^{-\beta} \sum_{l=0}^{k} C_{l}\left(\log \frac{C}{(1-t)}\right)^{-l}$, where $k=0,1,2, \cdots, h, h=[\alpha]$ and $C_{0}, C_{1}, \cdots, C_{h}$ are some constants, and

(iii) $Q_{\alpha}(t)=G_{\alpha, \beta}^{[h]}(t)$.

As necessary, the value of the functions at either of the end points of $[0,1]$ is defined by the corresponding one-sided limit. Thus with $\beta>1$ all the requirements in (1)-(4) on $q_{\delta}$ are met with $\delta=\alpha$ and

$$
q_{\alpha}(t)=G_{\alpha, \beta}^{\prime}(1-t), \quad 0 \leq t<1 .
$$

This complete a proof of the theorem. 
Remark 4.3.. 1. Bosanquet and Kestelman [3] proved that in the case $\alpha=1$ of Theorem B of Bosanquet, the summability $|C, \gamma|, \gamma>1$, may not, in general, be replaced by summability $|C, 1|$. One believes that here again, in the case $\alpha=1$, the summability $|\alpha, \beta|, \alpha=1, \beta>1$, in general, may not be replaced by the summability $|\alpha, \beta|, \alpha=1, \beta=1$. That is, the summability $|\alpha, \beta|, \alpha=1$, $\beta=1$, of Fourier series is not a local property of the generating function of the series (cf. Chandra and Karanjgaokar [7]).

2. One may note that results on summability factors for Fourier series for the summability $|\alpha, \beta|$, as given by Patra [14] may also now be deduced as very special cases of Theorem 2.4 on Nevanlinna summability as proved in [8].

\section{References}

[1] L. S. Bosanquet, Note on the absolute summability of a Fourier series, J. London Math. Soc., 11 (1936), 11-15.

[2] L. S. Bosanquet, The absolute Cesàro summability of a Fourier series, Proc. London Math. Soc., 41(2) (1936), 517-528.

[3] L. S. Bosanquet and H. Kestelman, The absolute convergence of series of integrals, Proc. London Math. Soc., 45(2) (1939), 88-97.

[4] L. S. Bosanquet and E. H. Linfoot, On the zero order summability of Fourier series, J. London Math. Soc., 6 (1931), 117-126.

[5] L. S. Bosanquet and E. H. Linfoot, Generalized means and the summability of Fourier series, Quart. J. Math. Oxford Ser., 2(2) (1931), 207-229.

[6] B. J. Boyer and L. I. Holder, A generalization of absolute Rieszian summability, Proc. Amer. Math. Soc., 14 (1963), 459-464.

[7] P. Chandra and V. Karanjgaokar, An aspect of local property of the Fourier series, (forthcoming: Indian J. Math.)

[8] G. D. Dikshit, Absolute Nevanlinna summability and Fourier series, J. Math. Anal. Appl., 248 (2000), 482-508.

[9] L. I. Holder, The absolute summability of Fourier series by the BosanquetLinfoot method, J. Math. Anal. Appl., 92 (1983), 381-396.

[10] A. F. Moursund, On a method of summation of Fourier series, Ann. of Math., 33(2) (1932), 773-784. 
[11] A. F. Moursund, On a method of summation of Fourier series, Ann. of Math., 34(2) (1933), 778-798.

[12] F. Nevanlinna, Über die Summation der Fourier'schen Reihen und Integrale, Översikt Finska Vetenskapps-Societetens Förhandlinger A, 64(3) (1921-1922).

[13] B. Patra, On the absolute zero order summability of a Fourier series and its allied series, Indian J. Pure and Appl. Math., 13 (1982), 785-794.

[14] B. Patra, On the absolute $(\alpha, \beta)$ summability of some series associated with a Fourier series and its allied series, Indian J. Pure and Appl. Math., 21 (1990), 530-543.

[15] B. K. Ray and M. Samal, Application of the absolute $N_{q}$-method to some series and integrals, J. Indian Math. Soc., 44 (1980), 217-236.

[16] M. Samal, On the absolute $N_{q}$-summability of some series associated with Fourier series, J. Indian Math. Soc., 50 (1986), 191-209. 
